\title{
Blood and cerebrospinal fluid markers in Parkinson's disease: current biomarker findings
}

\author{
This article was published in the following Dove Press journal: \\ Current Biomarker Findings \\ 17 December 2014 \\ Number of times this article has been viewed
}

\section{Claire Henchcliffe}

Department of Neurology, Weill Cornell Medical College, New York, NY, USA
Correspondence: Claire Henchcliffe Weill Cornell Medical College, Department of Neurology, 428 East 72nd Street, Suite 400, New York, NY 10022, USA

$\mathrm{Tel}+\mathrm{I} 2127462584$

Fax + I 2127468296

Email clh2007@med.cornell.edu
Abstract: Parkinson's disease (PD) is a common, progressive, and disabling neurodegenerative movement disorder. Signs and symptoms begin insidiously and overlap with other neurodegenerative conditions, making diagnosis challenging in early disease. Moreover, there are as yet no established biomarkers that will objectively measure disease progression, and this hampers efforts to obtain neuroprotective therapies. At present, diagnosis, measurement of progression, and response to therapeutic intervention rely almost exclusively upon clinical observation. There remains, therefore, a critical need for validated biomarkers. Recent advances in understanding the underlying pathophysiology of PD have aided in identifying strong candidate markers to assist in diagnosis and evaluate progression. Pathways that are disrupted in PD include protein misfolding, mitochondrial dysfunction and increased oxidative stress, dysregulated inflammatory processes, and altered gene expression. For example, $\alpha$-synuclein is a key contributor to PD pathology, in which misfolded and damaged $\alpha$-synuclein accumulates in neurons; this is now a leading candidate as a PD biomarker in cerebrospinal fluid. There is also recent evidence that markers of Alzheimer's disease may be "repurposed" to provide information on PD diagnosis, prognosis, and nonmotor symptoms, including cognitive dysfunction. At the same time, increasingly sophisticated bioinformatics technology allows an unbiased approach to biomarker identification, for example, using proteomic, transcriptomic, and metabolomic analysis. Such generally accessible blood or cerebrospinal fluid tests, if successful, will likely lead to significant improvements in PD diagnosis, management, and research.

Keywords: Parkinson's disease, neurodegeneration, $\alpha$-synuclein, oxidative stress, tau

\section{Background}

Parkinson's disease (PD) is a common, progressive, and disabling neurodegenerative disease affecting approximately $1 \%$ of people over 65 years of age. In the US, the combined direct and indirect costs of PD amount to approximately $\$ 25$ billion per year. Worldwide prevalence is predicted to at least double from 2005 to $2030^{1}$ and this will lead to increasing challenges to optimal health care. The cardinal clinical signs of PD are bradykinesia, rigidity, and rest tremor, affecting speech, hand coordination, gait, and balance. These are attributed for the most part to progressive degeneration of dopaminergic input from the substantia nigra within the midbrain to the striatum, ${ }^{2}$ thus disrupting complex downstream pathways critical for motor function. Molecular and chemical pathways involving dopamine have, therefore, been a priority as targets in biomarker development, with considerable success in initial studies using neuroimaging of the dopamine pathway. However, it is increasingly understood that PD pathology is

submit your manuscript $\mid$ www.dovepress.con 
more widespread, involving multiple complex biochemical, molecular, and cellular pathways, that exist both within the central nervous system as well as systemically.

Objective biomarkers for PD would serve to advance clinical care and research in several different ways. A first critical need for a PD biomarker is in diagnosis. PD diagnosis is still made upon clinical grounds, combining history and physical findings. Unfortunately, clinical diagnosis can be challenging, and PD presents insidiously and heterogeneously. Multiple studies have underlined the risk for diagnostic error. ${ }^{3,4}$ This is a particular risk in early disease when symptoms and signs are subtle and may overlap with other neurological disorders. ${ }^{5}$ A valid diagnostic biomarker for PD would not only improve diagnostic timeliness and accuracy, but would potentially define disease subtypes. ${ }^{6}$

A second critical need is to meet the challenge of measuring progression of the underlying neurodegenerative disease. Changes in disease status, for example in response to treatment or with disease progression over time, are still measured using clinical rating scales, in particular the Unified Parkinson's Disease Rating Scale (UPDRS). ${ }^{7}$ However, signs and symptoms are at least partially masked by symptomatic treatment, thus confounding clinical rating scale evaluations of PD progression. This has likely contributed to recent frustration over the difficulty translating promising preclinical and early phase clinical trial neuroprotection data into approved therapies. Hence, there is optimism that a biomarker incorporated as a clinical trial outcome measure, or "surrogate marker", would objectively and quantitatively track PD progression, opening the way to more sophisticated testing of potential neuroprotectants.

A third critical need is the ability to determine "target engagement" for any given intervention. This would be informative in making go/no go decisions during earlyphase drug development. It would also flesh out understanding of clinical trial results. For example, in the case of an unsuccessful clinical trial, if target engagement could be verified, it would prompt examination of whether the target itself is worth pursuing in drug development.

Biomarker development in PD has traditionally been focused on neuroimaging, which therefore holds a significant lead time. However, there is an emerging focus upon blood and cerebrospinal fluid (CSF)-based markers. ${ }^{8}$ These have a number of advantages, including accessibility, cost, greater variety of targets available for testing, and a generally lower burden on the patient. The availability of increasingly sophisticated technology has now opened up the possibilities for biofluid-based biomarker development. Moreover, breakthroughs in understanding the neuroscience and molecular genetics of PD have supported development of targeted approaches to biomarker development. This article describes the most relevant recent studies in biomarker development using CSF and blood components as biosamples, and examines potential future directions.

\section{Developing landscape of CSF and blood biomarkers for PD}

Advances in understanding the molecular and genetic underpinnings of PD have identified "key players" that may be targeted as potential biomarkers (Figure 1). These are components of pathways that lead to important processes in PD etiopathogenesis, including disrupted dopamine metabolism, aberrant protein aggregation, oxidative stress and mitochondrial dysfunction, inflammation, and transcriptional dysregulation. Moreover, the "omics" technologies have raised the possibility of unbiased identification of potential biomarkers. The following is not an exhaustive list of potential markers, but describes progress in identifying promising avenues based upon both targeted and untargeted approaches (for summary, see Table 1).

\section{Dopamine-based biomarkers}

Although dopamine deficiency in the nigrostriatal pathway is a primary feature of PD, and it would therefore seem intuitive that dopamine levels in biofluids might be altered, measuring dopamine directly in blood and CSF has proved disappointing. Older studies examined endogenous

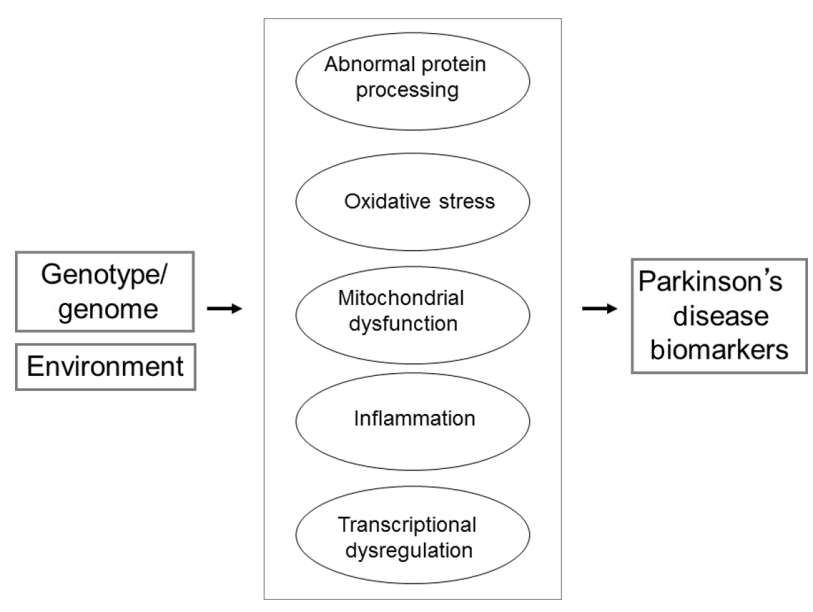

Figure I The central panel lists cellular pathways disrupted in the pathogenesis of PD that have been targeted for development of PD biomarkers. These abnormalities are thought to arise from a combination of genetic and environmental factors, as depicted on the left side of the panel.

Abbreviation: PD, Parkinson's disease. 
Table I Promising Parkinson's disease-associated biomarkers in blood and CSF

\begin{tabular}{|c|c|c|c|}
\hline & Blood & CSF & Comments \\
\hline \multicolumn{4}{|l|}{ Proteins and peptides } \\
\hline \multirow[t]{2}{*}{$\alpha$-synuclein (PARKI locus) } & Variable & $\downarrow$ & May correlate with worsened motor disability \\
\hline & $\uparrow$ antibodies & & Specific isoforms likely important \\
\hline DJ-I (PARK7 locus) & Variable & $\downarrow$ & May depend on specific isoform \\
\hline$A \beta_{1-42}$ & & $\downarrow$ & May correlate with cognition \\
\hline p-tau & & $\downarrow$ & May associate with postural instability-gait disorder subtype \\
\hline t-tau & & $\downarrow$ & May associate with worsened motor disability and cognition; rate of change \\
\hline & & & may correlate with progression \\
\hline ApoAl & & $\downarrow$ & \\
\hline IL-I0, RANTES & $\uparrow$ & & \\
\hline TNF- $\alpha$ & $\uparrow$ & $\uparrow$ & \\
\hline $\begin{array}{l}\text { Antineuronal antibodies, } \\
\text { antimelanin antibodies }\end{array}$ & & $\uparrow$ & \\
\hline \multirow[t]{4}{*}{ RNAs: transcriptome changes } & & & Proteasome subunit- $\alpha$ type- 2 , laminin $\beta-2$, aldehyde dehydrogenase I family- \\
\hline & & & member AI, histone cluster-I H3e; STI3; heat shock $70 \mathrm{kDa}$ protein $8, \mathrm{pl} 9$ \\
\hline & & & S-phase kinase-associated protein IA, huntingtin-interacting protein-2, I9S \\
\hline & & & proteasomal protein PSMC4, aldehyde dehydrogenase family I subfamily AI \\
\hline miRNAs & & & $\begin{array}{l}\downarrow \text { miR-339-5p, miR-30c, miR-I48b, } \uparrow \text { miR-223, miR-324-3p, miR-24; } \\
\downarrow \text { miR-29a, miR-29c, miR-19a, miR-19b }\end{array}$ \\
\hline \multicolumn{4}{|l|}{ Lipid-based } \\
\hline $\begin{array}{l}\text { Malondialdehyde, } \mathrm{F}(2)- \\
\text { isoprostanes, cholesterol } \\
\text { oxidation products }\end{array}$ & $\uparrow$ & & Reflects oxidative damage \\
\hline \multicolumn{4}{|l|}{ Small molecules } \\
\hline HVA & & $\uparrow$ xanthine/HVA & May associate with PD progression \\
\hline $80 \mathrm{HdG}$ & $\uparrow$ & $\uparrow$ & Reflects oxidative DNA damage \\
\hline Urate & $\uparrow$ & $\uparrow$ & May associate with progression \\
\hline $\begin{array}{l}\text { Hypoxanthine, hypoxanthine/ } \\
\text { xanthine ratio, and } \\
\text { xanthosine/xanthine ratio }\end{array}$ & $\downarrow$ & & \\
\hline
\end{tabular}

Abbreviations: $A \beta_{1-42}, \beta$ amyloid 42 amino acid isoform; ApoAI, apolipoprotein AI; CRP, C-reactive protein; CSF, cerebrospinal fluid; HVA, homovanillic acid; IL-6, interleukin-6; IL-10, interleukin I0; MCP-I, monocyte chemotactic protein I; miRNAs, micro RNAs; p-tau, phosphorylated tau; RANTES, Regulated on Activation, Normal T cell Expressed and Secreted chemokine; t-tau, total tau; TNF- $\alpha$, tumor necrosis factor alpha; 8OHdG, 8-hydroxy-2-deoxyguanosine.

tetrahydroquinolones reflecting dopamine metabolism, and salsolinol was suggested in one study to be associated with PD dementia. ${ }^{9}$ Altered levels of dopamine metabolites and related compounds have been more recently demonstrated. Homovanillic acid (HVA) is a product of dopamine breakdown. A recent study employing metabolomic analysis compared levels of the purine compounds, xanthine and HVA. The CSF ratio of xanthine/HVA was demonstrated to differ significantly between 217 patients with PD and 26 controls, with a higher value in PD compared with controls. ${ }^{10} \mathrm{PD}$ participants were not taking anti-PD medications, thus the difference could not be attributed to medication effects. Moreover, the xanthine/HVA ratio increased with time in a longitudinal element of the study, suggesting that it is a possible marker of both state and trait. Not only is there disruption of dopamine production in $\mathrm{PD}$, but there is also evidence that its metabolism may be disrupted. In particular, the enzyme responsible for dopamine breakdown, monoamine oxidase B, has been found to be increased in plasma samples collected from patients with PD. ${ }^{11}$

\section{$\alpha$-synuclein, a key component in $P D$ pathogenesis}

Protein damage and misfolding are key to $\mathrm{PD}$ pathogenesis. These processes are important in formation of the structures that comprise the hallmark pathology of PD, ie, Lewy bodies (Figure 2). The $\alpha$-synuclein gene was the first locus identified in which specific mutations result in Mendelian inheritance of PD (PARK1 gene locus), and although these mutations are rare, identification of $\alpha$-synuclein protein as a contributor to PD pathogenesis revolutionized understanding of this process. Importantly, these pathological changes may occur in the "premotor" phase of PD, therefore also making $\alpha$-synuclein a strong candidate for monitoring PD both before and after motor symptoms arise. 


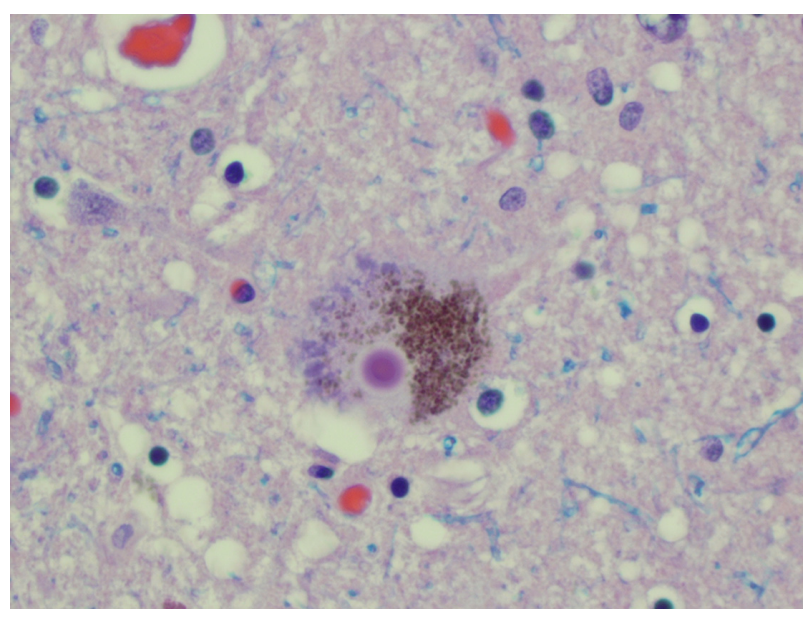

Figure 2 Photomicrograph of a hematoxylin-eosin-stained section taken from the substantia nigra pars compacta at autopsy of an individual with PD. A dopaminergic neuron in the center of the field contains a densely stained cytoplasmic Lewy body. Note: This article was published in Handbook of the Neuroscience of Aging. 2009, Schwarz C, Henchcliffe C. Parkinsonian syndromes. In: Hof P, Mobbs C, Editors. 44I-447. Copyright (C2009 Academic Press. ${ }^{2}$

$\alpha$-synuclein is primarily intracellular, but is detectable in CSF $^{12}$ and plasma, ${ }^{13-15}$ and decreased CSF $\alpha$-synuclein levels in PD have now been demonstrated in a number of studies. ${ }^{12,16-23}$ Two of these studies examined patients with early PD, making it unlikely that results are simply associated with anti-PD medication effects..$^{22,23}$

These studies have raised hopes that $\alpha$-synuclein could be a first diagnostic marker for PD. However, specificity remains to be better defined. One study found decreased CSF $\alpha$-synuclein in Alzheimer's disease (AD) versus controls, leading the investigators to postulate that it is a marker of loss of synapses in general. ${ }^{16}$ Similarly, decreased CSF $\alpha$-synuclein has been seen in dementia with Lewy bodies and multiple system atrophy. ${ }^{21}$ Whether CSF $\alpha$-synuclein levels will objectively track PD progression remains to be determined, but there are some hopeful preliminary data. The Parkinson's Progression Markers Initiative (PPMI, ClinicalTrials.gov identifier NCT01141023) enrolled 400 participants with early PD, and baseline data showed that a decreased CSF $\alpha$-synuclein concentration was associated with increased motor severity in PD. ${ }^{22}$ This will now need to be tested longitudinally. Interestingly, in a post hoc analysis of CSF $\alpha$-synuclein levels in the DATATOP (Deprenyl And Tocopherol Antioxidative Therapy Of Parkinsonism) study, lower levels were associated with cognitive decline longitudinally. ${ }^{24}$

An important issue to consider is whether a combination of protein measures incorporating $\alpha$-synuclein might constitute a superior biomarker. In one study, a panel of seven proteins comprising $\alpha$-synuclein, DJ-1, total tau (t-tau), phosphorylated tau ( $\mathrm{p}$-tau), fractalkine, Flt3 ligand, and
$\mathrm{A} \beta_{1-42}$ differed in CSF samples from PD when compared with normal controls, $\mathrm{AD}$, and multiple system atrophy. ${ }^{25}$

Although blood is more accessible than CSF in the clinic, developing blood-based measures of $\alpha$-synuclein has proven more complicated. In plasma, total ${ }^{26}$ and oligomeric ${ }^{13}$ $\alpha$-synuclein has been increased in PD, but this has not been replicated in other studies. ${ }^{15,27,28}$ It has been proposed that variations in sample collection and possibly hemolysis might lead to variability in measures, as the vast majority of $\alpha$-synuclein is present in red blood cells.

Finally, anti- $\alpha$-synuclein antibodies have been detected in association with PD, and are worthy of consideration as potential markers of disease. These specific antibodies have been detected in one study in approximately $50 \%$ of individuals with sporadic PD, $90 \%$ of individuals with familial PD, and $30 \%$ of control cases. ${ }^{29}$ Although another study failed to confirm the finding, ${ }^{30}$ a recent study examined autoantibodies to the monomeric form $\alpha$-synuclein and found an increase in these antibodies in serum in PD. ${ }^{31}$

There is, consequently, a need for further studies to better delineate the potential role for $\alpha$-synuclein as a PD biomarker. It may be that the specific forms of $\alpha$-synuclein need to be investigated individually, for example, the ratio of nitrated to non-nitrated $\alpha$-synuclein as suggested by Sharma et $a l,{ }^{8}$ to make sense of the variability in findings to date.

\section{Markers of oxidative stress and mitochondrial dysfunction}

Mitochondrial dysfunction has been widely documented in PD, with defects in oxidative phosphorylation, calcium homeostasis, and biogenesis. ${ }^{32,33}$ Early studies determined deficits in individual complexes in the electron transport chain, for example, mitochondrial complex I in platelets. ${ }^{34}$ Moreover, Charnoly bodies, abnormally aggregated mitochondrial membranes, are found in degenerating neurons. It has been suggested that these structures as well as components affecting their formation (such as metallothioneins) could serve as PD biomarkers. ${ }^{8}$ Increased oxidative stress, with damage to DNA, RNA, proteins, and lipids, are closely linked since mitochondria are the major cellular sources of damaging free radicals. Markers that would reflect key changes in these interrelated processes have therefore been of great interest. Altered redox status in PD has been demonstrated in studies of two potent antioxidants, coenzyme Q10 in blood ${ }^{35,36}$ and $\mathrm{CSF}^{37}$ and glutathione in plasma samples. ${ }^{38}$ Oxidative damage to DNA has been demonstrated in PD by detection of elevated levels of 8-hydroxy-2-deoxyguanosine, 
a compound formed by direct oxidative damage to nucleosides in CSF, plasma, and urine..$^{39,40}$ Markers of oxidative damage to lipids are similarly elevated. Malondialdehyde, formed as reactive oxygen species degrade polyunsaturated lipids, was found to be significantly increased in plasma levels in $\mathrm{PD}^{38,41}$ and plasma $\mathrm{F}(2)$-isoprostanes (a fatty acid peroxidation product) and cholesterol oxidation products have been found to be elevated in PD. ${ }^{42}$

Urate is included in this section since it is a potent antioxidant, as well as a metal chelator capable of binding iron. ${ }^{43-45}$ It is the end product of purine breakdown, with precursors including hypoxanthine and xanthine, which have also been examined as possible biomarkers of PD. Multiple studies have found the urate concentration in serum and CSF to be inversely associated with occurrence of PD, particularly in men. ${ }^{46,47}$ Moreover, higher serum and CSF urate concentrations have been associated with slower PD progression. ${ }^{47,48}$ Hypoxanthine, the hypoxanthine/xanthine ratio, and the xanthosine/xanthine ratio were also found to be reduced in a study of PD versus controls. ${ }^{49}$ As noted earlier, the xanthine/ HVA ratio is perturbed in PD and changed with disease progression in one study. ${ }^{10}$ This deserves further study in longitudinal cohorts to verify the potential of this ratio as a potential marker of progression.

\section{Markers of disrupted inflammatory pathways}

There are multiple lines of evidence demonstrating the presence of inflammation in PD. Microglial activation is observed in autopsy specimens of brain tissue in PD, and neuroimaging using the peripheral benzodiazepine receptor ligand PK11195 is consistent with microglial activation in living PD patients. ${ }^{33}$ It is therefore not surprising that several studies have now described alterations in inflammatory pathways in blood and CSF in PD. Increased levels of interleukin-10 and the chemokine RANTES (Regulated on Activation, Normal T cell Expressed and Secreted) have been found in serum collected from individuals with PD compared with controls. ${ }^{50}$ Elevated tumor necrosis factor (TNF)- $\alpha$ levels have been detected in association with PD in both plasma and $\mathrm{CSF}^{51,52}$ Abnormal antibody expression associated with PD includes CSF antineuronal antibodies, ${ }^{53}$ anti- $\alpha$-synuclein antibodies, ${ }^{29,31}$ and antimelanin antibodies. ${ }^{54}$

Although these examples are associated with PD diagnosed on the basis of motor symptoms, a proinflammatory state seems to be associated with certain nonmotor symptoms of PD. ${ }^{55}$ Worse scores on psychometric testing have been associated with increased soluble TNF receptor levels in plasma. ${ }^{56}$ Measures of CSF markers, including C-reactive protein, interleukin 6 , TNF- $\alpha$, interferon 10 , monocyte chemotactic protein 1 , and macrophage inflammatory protein $1-\beta$, in individuals with PD found inflammatory markers to be significantly associated with more severe depression, anxiety, fatigue, and cognition. ${ }^{57}$ These independent lines of evidence therefore support inflammation as an important pathway in $\mathrm{PD}$, in which perturbations might serve as biomarkers with different purposes.

\section{PD genetics and biomarker development}

Major advances in understanding the genetic underpinnings of PD have identified a number of proteins that are key in cellular pathways that are altered. As noted earlier, $\alpha$-synuclein is now a leading target for development as a PD biomarker. However, other PD-associated genes are now under intensive investigation as biomarker candidates.

One such gene is $D J-1$ (PARK7 locus). Mutations in the $D J-1$ gene are a rare cause of autosomal recessive forms of $\mathrm{PD}$, and the corresponding protein is detected in both blood and CSF. As with $\alpha$-synuclein, in blood the overwhelming majority of the protein is contained within blood cells and platelets, making sample preparation demanding, and with variable hemolysis or residual platelets in samples leading to a potential source of noise. ${ }^{27}$ This may account for contradictory results published in plasma. ${ }^{58,59} \mathrm{CSF}$ measures may therefore turn out to be more robust. A recent study of DJ-1 protein described decreased levels in CSF samples from study participants with PD compared with healthy volunteers. With findings of $90 \%$ sensitivity and $70 \%$ specificity, performance characteristics were encouraging. ${ }^{17}$ Moreover, in this cohort, CSF $\alpha$-synuclein and DJ-1 levels correlated. Once again, however, findings have been contradicted by other groups: a previous study found increased rather than decreased CSF DJ-1 levels associated with a PD diagnosis, particularly in early stages. ${ }^{60}$ Moreover, although levels may be associated with the diagnosis of PD, DJ-1 does not seem on the basis of current studies to be a marker of disease severity. ${ }^{25,27}$ As with $\alpha$-synuclein, however, DJ-1 exists in multiple forms. It will likely be important to define which will be most helpful as biomarkers. In a study of seven DJ-1 isoforms detected in blood, those with post-translational 4-hydroxy-2-nonenal modifications were altered in late PD. ${ }^{61}$ In an independent study, DJ-1 oxidized on cysteine-106 specifically was found to be elevated in red blood cells in PD. ${ }^{62}$ Initial results are therefore somewhat encouraging, with the caveat that careful consideration of 
the various isoforms of DJ-1, including post-translational modifications, will be necessary.

\section{AD biomarkers and PD: $\beta$-amyloid and tau}

$\beta$-amyloid $(A \beta)$ and tau proteins are being intensively studied in AD, and measures of the 42 amino acid isoform of $\beta$-amyloid $\left(A \beta_{1-42}\right)$ and t-tau and $p$-tau concentrations in $\mathrm{CSF}$ are now included in AD diagnostic criteria. There is also evidence that they predict AD risk. ${ }^{63}$ However, based upon strong recent evidence, these proteins may turn out to be at least as important for use as PD biomarkers. In ventricular CSF from postmortem PD cases compared with controls, it was found that the $\mathrm{p}$-tau/A $\beta_{1-42}$ ratio and apolipoprotein A1 levels were significantly associated with the diagnosis of PD. ${ }^{64}$

The potential for development of amyloid and tau proteins as PD biomarkers in CSF is further supported by several independent studies. The PPMI is an international longitudinal observational study following 400 subjects with early PD who are drug-naïve, and healthy matched control subjects are also enrolled for comparison. ${ }^{65}$ In this PPMI cohort, lower $A \beta_{1-42}$ and p-tau CSF levels were significantly associated with PD. ${ }^{22}$ Moreover, decreased t-tau levels were found to be associated with increased severity of motor symptoms. Interestingly, lower $A \beta_{1-42}$ and p-tau were both associated with the subset of PD subjects with postural instability-gait disorder rather than those categorized as having tremor-predominant PD. Since tremor predominance in symptom pattern has been associated with better outcome than postural instability-gait disorder in multiple independent studies, this raises the possibility that lower CSF levels of $A \beta_{1-42}$ and $\mathrm{p}$-tau proteins might be predictive of a worse prognosis.

The DATATOP study ${ }^{66}$ was designed to test whether deprenyl (selegiline) and/or $\alpha$-tocopherol would slow PD progression, but has also proved invaluable for data mining, given that clinical data, blood samples, and CSF were collected in this large PD cohort over many years. Recent analysis of 403 CSF samples from the DATATOP study found that $A \beta_{1-42}$ levels had a weak negative correlation with total UPDRS scores in early PD, and baseline $\mathrm{p}$-tau/t-tau and $\mathrm{p}$-tau/ $\mathrm{A} \beta_{1-42}$ ratios were found to have a negative correlation with progression as measured by the rate of change of UPDRS scores. ${ }^{67}$ A significant (although weak) positive correlation was also observed between disease progression as measured by change in UPDRS score and the rate of change in t-tau level and $t$-tau $/ A \beta_{1-42}$ ratio. As in $A D$, these proteins may also prove to be markers that reflect cognitive decline. CSF levels of $\mathrm{A} \beta_{1-42}$ and t-tau proteins, as well as $\mathrm{A} \beta_{1-42} /$ total tau ratio, have been described to correlate with multiple scores of cognition, including Logical Memory (delayed), Category Fluency, Digit Symbol, and Trails B minus A, in 22 nondemented individuals with PD. ${ }^{68}$

Finally, combinations of $A \beta_{1-42}$ measures with other proteins may also prove worthy of further investigation. The CSF complement $3 / \mathrm{A} \beta_{1-42}$ ratio and factor $\mathrm{H} / \mathrm{A} \beta_{1-42}$ ratio $^{69}$ have both been found to be associated with PD motor severity, cognitive impairment, and dementia. The fractalkine/A $\beta_{1-42}$ ratio in a cross-sectional study of PD CSF samples correlated with disease severity, and also with disease progression in samples collected longitudinally in this study cohort. ${ }^{25}$ As in $A D$, reduced $A \beta_{1-42}$ levels have been associated with cognitive decline in CSF samples from PD patients. ${ }^{70}$

\section{Omics technology and PD biomarker discovery}

In contrast with the targeted markers discussed above, the omics approach has been applied to PD biomarker development for unbiased and systematic evaluation of patterns of variation in RNAs (transcriptomics), proteins (proteomics), lipids (lipidomics), or small molecules (metabolomics). Omics combines experimentation and mathematical modeling, making use of large datasets acquired from pools of biological molecules that may be present in blood, CSF, urine, or tissue. ${ }^{71}$ Use of powerful mass spectrometry, nuclear magnetic resonance, and related techniques has been central to developing this field. For example, mass spectrometry is capable of high sensitivity detection and of quantifying thousands of molecular species. ${ }^{72}$ The approach provides the possibility to define combinations or "fingerprints" of molecular species that may serve as disease biomarkers. Although early in application in PD, omics analyses have already demonstrated promise in neurodegenerative disorders, ${ }^{73}$ and it is hoped that identification of one or more species (protein, peptide, RNA, or small molecule), or a composite "fingerprint" will prove feasible that could be of use in diagnosis, measuring disease progression, or determining a response to a specific intervention.

Multiple studies employing transcriptomic analysis have demonstrated dysregulated gene expression in PD brain tissue. ${ }^{7-76}$ In some cases, such differences have served as a basis for identification of potential biomarkers in blood. For example, based upon differential expression in brain tissue, 12 selected candidate transcripts were subjected to analysis using quantitative reverse transcription polymerase chain 
reaction (RT-PCR) in blood samples. ${ }^{77}$ The investigators demonstrated that a measure comprising transcript levels for proteasome subunit- $\alpha$ type- 2 , laminin $\beta-2$, aldehyde dehydrogenase 1 family-member A1, and histone cluster-1 H3e, was capable of achieving over $80 \%$ sensitivity and specificity for association with the diagnosis of PD. In a case-control study of 66 subjects, a combination of eight gene transcripts were associated with risk of PD, and results were subsequently validated in an independent sample set. ${ }^{78}$ It is intriguing that one of these transcripts encodes the co-chaperone ST13, a stabilizer of heat shock protein 70 , which is known to modify $\alpha$-synuclein misfolding and toxicity. Other transcripts have been identified by omics analysis as potential candidates for PD biomarkers. Five transcripts were found to be altered in blood in early PD, encoding the following proteins: heat shock $70 \mathrm{kDa}$ protein 8; p19 S-phase kinase-associated protein 1A; huntingtin-interacting protein-2; 19S proteasomal protein PSMC4; and aldehyde dehydrogenase family 1 subfamily A $1 .{ }^{79}$ As predictors of PD diagnosis, the panel had high sensitivity and specificity.

There is growing interest in proteomic analysis to identify PD biomarkers. In an initial study of human substantia nigra pars compacta tissue, two-dimensional gel electrophoresis identified nine specific proteins that were differentially expressed in PD when compared with healthy control subjects ${ }^{80}$ and a further analysis increased this number, identifying 221 differentially expressed proteins. ${ }^{81}$ Moreover, a rationale for altered expression in PD for many of these proteins can be postulated. For example, differential expression of glutathione S-transferase and other genes involved in glutathione metabolism might relate to the importance of oxidative stress in PD pathogenesis. Altered expression of glial and related proteins, such as GFAP, GMFB, galectin-1, and sorcin, might reflect known changes in activity and presence of glial cells as PD pathology accrues. The challenge of translating this technology to fluid-based biosamples has now been met. Using a different proteomic platform, over 1,500 CSF proteins were analyzed using isobaric Tagging for Relative and Absolute Protein Quantification (iTRAQ) followed by mass spectrometry. ${ }^{82}$ Samples were compared between PD (based upon clinical diagnosis), dementia with Lewy bodies (autopsy confirmed), AD (autopsy confirmed), and healthy controls. Of the 1,500 proteins, 72 were differentially expressed in $P D$, and specific combinations of proteins were identified that distinguished the different groups. A study of CSF proteins compared surface-enhanced laser desorption/ionization time-of-flight mass spectrometry in 56 subjects with PD, 24 control subjects, as well as samples from individuals with the clinically closely related disorders of multiple system atrophy, progressive supranuclear palsy, and corticobasal degeneration. CSF levels of ubiquitin, beta2-microglobulin, and two secretogranin 1 fragments differentiated PD from atypical parkinsonism, ${ }^{83}$ although they did not differentiate PD from controls. Using matrix-assisted laser desorption/ionization time-of-flight mass spectrometry (MALDI-TOF-MS), other protein species have been identified that are differentially expressed between PD and controls, ${ }^{84-86}$ and recent analysis of proteins captured by magnetic bead-based weak cation exchange followed by MALDI-TOF-MS in a blinded analysis identified a combination of five biomarkers discriminating PD and healthy controls with $85 \%$ sensitivity and $70 \%$ specificity. ${ }^{87}$ Finally, use of two-dimensional gel electrophoresis and mass spectrometry has so far yielded encouraging data supporting the possibility of developing a diagnostic biomarker on the basis of a proteomic fingerprint. ${ }^{88,89}$

The potential for defining fingerprints of combinations of lipids is now being explored. A recent investigation of lipids isolated from blood samples of healthy adults, with cognition measured in the normal range, determined a set of ten lipid species predicting development of amnestic mild cognitive impairment or AD. ${ }^{90}$ Since overlapping cellular processes, including effects upon cell membrane integrity, may be at play in PD, this approach will need to be thoroughly evaluated.

Metabolomic profiling, which examines arrays of low molecular weight species, has also proved promising in identifying PD biomarkers. In plasma, metabolomic analysis using high-performance liquid chromatography with electrochemical coulometric array detection for quantitative assessment of approximately 2,000 small analytes has successfully distinguished subjects with PD versus healthy controls. ${ }^{39}$ In plasma samples from 15 drug-naïve PD patients and 25 controls, metabolomic analysis was capable of deriving fingerprints that completely distinguished the two groups $(P<0.01)$. From these fingerprints, it was then possible to choose a subset of analytes that had the most significant contribution to differences measured. This same analyte subset was shown to be capable of distinguishing individuals with PD treated with carbidopa/levodopa versus controls, as well as individuals with PD treated with both carbidopa/ levodopa and dopamine agonists versus controls $(P<0.01)$. Using the same platform for metabolomic analysis, in a further study of individuals with idiopathic PD and LRRK2associated PD, these two groups could be distinguished from each other, and also from controls. ${ }^{49}$ It is encouraging that when examining levels of individual analytes, urate (see the 
"Markers of oxidative stress and mitochondrial dysfunction" section) was found to be lower in idiopathic and LRRK2 PD than control samples. ${ }^{39,49}$ A smaller decrease was measured in LRRK2 nonmanifesting carriers, and, although not statistically significant, it suggests that urate needs to be further investigated in "at risk" individuals. ${ }^{49}$ Additionally, measures of dopamine and purine metabolites (hypoxanthine, HVA/ xanthine, xanthosine/xanthine) have been demonstrated to be significantly reduced in PD subjects not taking anti-PD medication when compared with controls. ${ }^{49}$ As noted earlier, use of a metabolomics analysis platform also detected abnormalities in the HVA/xanthine concentration ratio in PD that changed with disease progression over a 2-year period..$^{10,91}$

\section{MicroRNA profiling}

MicroRNAs (miRNAs) are small noncoding RNAs, and are thought to play a pivotal role in regulating gene expression at the post-transcriptional level, affecting transcript stability and translation of multiple genes, including $\alpha$-synuclein. A number of studies have demonstrated differences in specific miRNAs of brain tissue in PD versus healthy controls, but more recently key differences have been detected in circulating miRNAs, making their profiling in peripheral blood a promising approach for biomarker development. Vallelunga et al recently reported a set of nine specific miRNAs out of approximately 750 examined that could distinguish PD from controls and subjects with multiple system atrophy. ${ }^{92}$ A nonoverlapping set of four specific miRNAs was found in an independent study to be downregulated in PD, including the genetic $L R R K 2$-associated form of PD, versus controls. ${ }^{93}$

\section{Conclusion and future directions}

There is a critical need for validated biomarkers in the clinic and in PD research. The use of fluid-based biomarkers has many advantages over neuroimaging. Testing blood or CSF often places less of a burden on the patient, is easier to perform and therefore not limited to selected medical facilities, and is usually cheaper. Moreover, the use of fluid-based biomarkers provides access to a greater variety of molecular processes that may be at play in PD. At this time, obtaining biomarkers based upon blood or CSF remains in the early phase of development for PD. Many of the findings described in this review still require validation. However, there are now a small number of candidate fluid-based markers that are ready for incorporation into testing of target engagement, such as CSF urate level after administration of inosine. ${ }^{94}$ Early biomarker studies described in this review have focused largely on cross-sectional studies comparing PD with healthy or disease controls, and this will identify potential markers for diagnosis. However, longitudinal studies will be paramount to identify markers of disease progression. It will also be important to define how early in the disease process any identified diagnostic marker will be useful. There is now hope to identify PD in its premotor phase, and testing fluid-based biomarkers in at-risk cohorts will be critical. It may be that a biomarker useful in the early stages of PD will be different than one useful in advanced PD, and it is therefore doubtful that a single biomarker will suffice in a disorder as complex as PD.

With rapid development of biomarker technologies, the availability of resources for discovery and validation is now vital. As noted above, the longitudinal observational PPMI study is an outstanding example of an international collaborative initiative that is collecting biosamples in early PD, as well as clinical and neuroimaging data, under rigorous conditions. ${ }^{65}$ The BioFIND study (Fox Investigation for New Discovery of Biomarkers in Parkinson's disease, ClinicalTrials.gov identifier NCT01705327) is ongoing at the time of writing, and will similarly provide a platform for discovery and validation of biomarkers in a welldefined cohort of individuals with moderate-to-advanced PD as well as control subjects for comparison. ${ }^{95}$

Development of blood-based and CSF-based biomarkers is now a high priority. Validated biomarkers hold the promise of improving diagnostic accuracy, accurately measuring disease progression, and objectively evaluating the molecular, biochemical, or neurophysiological impact of a given intervention. Ultimately, the hope is that biomarkers discovered in studies of PD will be applicable in premotor $\mathrm{PD}$, ie, to facilitate development of neuroprotective and/or neuropreventive interventions in at-risk populations of patients who have not yet manifested motor symptoms of PD.

\section{Disclosure}

$\mathrm{CH}$ receives research funding from the Solomon Family Foundation, the CV Starr Foundation, the Parkinson Disease Foundation, National Institute of Neurological Disorders and Stroke (NINDS), Biogen, and the Michael J Fox Foundation.

\section{References}

1. Dorsey ER, Constantinescu R, Thompson JP, et al. Projected number of people with Parkinson disease in the most populous nations, 2005 through 2030. Neurology. 2007;68(5):384-386.

2. Schwarz C, Henchcliffe C. Parkinsonian syndromes. In: Hof P, Mobbs C, editors. Handbook of the Neuroscience of Aging. Academic Press; 2009:441-447.

3. Hughes AJ, Daniel SE, Kilford L, Lees AJ. Accuracy of clinical diagnosis of idiopathic Parkinson's disease: a clinico-pathological study of 100 cases. J Neurol Neurosurg Psychiatry. 1992;55(3):181-184.

4. Adler CH, Beach TG, Hentz JG, et al. Low clinical diagnostic accuracy of early vs advanced Parkinson disease: clinicopathologic study. Neurology. 2014;83(5):406-412. 
5. Marshall VL, Reininger CB, Marquardt M, et al. Parkinson's disease is overdiagnosed clinically at baseline in diagnostically uncertain cases: a 3-year European multicenter study with repeat [123I]FP-CIT SPECT. Mov Disord. 2009;24(4):500-508.

6. Henchcliffe C, Dodel R, Beal MF. Biomarkers of Parkinson's disease and dementia with Lewy bodies. Prog Neurobiol. 2011;95(4):601-613.

7. Fahn S, Elton R. The UPDRS development committee. In: Fahn S, Marsden CD, Calne D, Goldstein M, editors. Recent Developments in Parkinson's Disease. Florham Park, NJ, USA: MacMillan Healthcare Information; 1987.

8. Sharma S, Moon CS, Khogali A, et al. Biomarkers in Parkinson's disease (recent update). Neurochem Int. 2013;63:201-229.

9. Antkiewicz-Michaluk L, Krygowska-Wajs A, Szczudlik A, Romańska I, Vetulani J. Increase in salsolinol level in the cerebrospinal fluid of parkinsonian patients is related to dementia: advantage of a new highperformance liquid chromatography methodology. Biol Psychiatry. 1997;42(6):514-518.

10. LeWitt P, Schultz L, Auinger P, Lu M; the Parkinson Study Group. CSF xanthine, homovanillic acid, and their ratio as biomarkers of Parkinson's disease. Brain Res. 2011;1408:88-97.

11. Zhou G, Miura Y, Shoji H, Yamada S, Matsuishi T. Platelet monoamine oxidase B and plasma beta-phenylethylamine in Parkinson's disease. J Neurol Neurosurg Psychiatry. 2001;70(2):229-231.

12. Tokuda T, Salem SA, Allsop D, et al. Decreased alpha-synuclein in cerebrospinal fluid of aged individuals and subjects with Parkinson's disease. Biochem Biophys Res Commun. 2006;349(1):162-166.

13. El-Agnaf OM, Salem SA, Paleologou KE, et al. Detection of oligomeric forms of alpha-synuclein protein in human plasma as a potential biomarker for Parkinson's disease. FASEB J. 2006;20(3):419-425.

14. Tinsley RB, Kotschet K, Modesto D, et al. Sensitive and specific detection of alpha-synuclein in human plasma. J Neurosci Res. 2010;88(12): 2693-2700.

15. Foulds PG, Mitchell JD, Parker A, et al. Phosphorylated alpha-synuclein can be detected in blood plasma and is potentially a useful biomarker for Parkinson's disease. FASEB J. 2011;25(12):4127-4137.

16. Ohrfelt A, Grognet P, Andreasen N, et al. Cerebrospinal fluid alphasynuclein in neurodegenerative disorders - a marker of synapse loss? Neurosci Lett. 2009;450(3):332-335.

17. Hong Z, Shi M, Chung K, et al. DJ-1 and alpha-synuclein in human cerebrospinal fluid as biomarkers of Parkinson's disease. Brain. 2010 133(3):713-726.

18. Waragai M, Sekiyama K, Sekigawa A, Takamatsu Y, Fujita M, Hashimoto M. alpha-Synuclein and DJ-1 as potential biological fluid biomarkers for Parkinson's disease. Int J Mol Sci. 2010;11(11): 4257-4266.

19. Mollenhauer B, Locascio JJ, Schulz-Schaeffer W, Sixel-Döring F, Trenkwalder C, Schlossmacher MG. alpha-Synuclein and tau concentrations in cerebrospinal fluid of patients presenting with parkinsonism: a cohort study. Lancet Neurol. 2011;10(3):230-240.

20. Wennström M, Surova Y, Hall S, al. Low CSF levels of both alphasynuclein and the alpha-synuclein cleaving enzyme neurosin in patients with synucleinopathy. PLoS One. 2013;8(1):e53250.

21. Hall S, Öhrfelt A, Constantinescu R, et al. Accuracy of a panel of 5 cerebrospinal fluid biomarkers in the differential diagnosis of patients with dementia and/or parkinsonian disorders. Arch Neurol. 2012;69(11): $1445-1452$.

22. Kang JH, Irwin DJ, Chen-Plotkin AS, et al. Association of cerebrospinal fluid beta-amyloid 1-42, T-tau, P-tau 181, and alpha-synuclein levels with clinical features of drug-naive patients with early Parkinson disease. JAMA Neurol. 2013;70(10):1277-1287.

23. Mollenhauer B, Trautmann E, Taylor P, et al. Total CSF alpha-synuclein is lower in de novo Parkinson patients than in healthy subjects. Neurosci Lett. 2013;532:44-48.

24. Stewart T, Liu C, Ginghina C, et al; Parkinson Study Group DATATOP Investigators. Cerebrospinal fluid alpha-synuclein predicts cognitive decline in Parkinson disease progression in the DATATOP cohort. Am J Pathol. 2014;184(4):966-975.
25. Shi M, Bradner J, Hancock AM, et al. Cerebrospinal fluid biomarkers for Parkinson disease diagnosis and progression. Ann Neurol. 2011;69(3): $570-580$.

26. Lee PH, Lee G, Park HJ, Bang OY, Joo IS, Huh K. The plasma alphasynuclein levels in patients with Parkinson's disease and multiple system atrophy. J Neural Transm. 2006;113(10):1435-1439.

27. Shi M, Zabetian CP, Hancock AM, et al. Significance and confounders of peripheral DJ-1 and alpha-synuclein in Parkinson's disease. Neurosci Lett. 2010;480(1):78-82.

28. Li QX, Mok SS, Laughton KM, et al. Plasma alpha-synuclein is decreased in subjects with Parkinson's disease. Exp Neurol. 2007; 204(2):583-588

29. Papachroni KK, Ninkina N, Papapanagiotou A, et al. Autoantibodies to alpha-synuclein in inherited Parkinson's disease. J Neurochem. 2007;101(3):749-756.

30. Besong-Agbo D, Wolf E, Jessen F, et al. Naturally occurring alphasynuclein autoantibody levels are lower in patients with Parkinson disease. Neurology. 2013;80(2):169-175.

31. Yanamandra K, Gruden MA, Casaite V, Meskys R, Forsgren L, Morozova-Roche LA. Alpha-synuclein reactive antibodies as diagnostic biomarkers in blood sera of Parkinson's disease patients. PLoS One. 2011;6(4):e18513

32. Henchcliffe C, Beal MF. Mitochondrial biology and oxidative stress in Parkinson disease pathogenesis. Nat Clin Pract Neurol. 2008;4(11) 600-609.

33. Hauser DN, Hastings TG. Mitochondrial dysfunction and oxidative stress in Parkinson's disease and monogenic parkinsonism. Neurobiol Dis. 2013;51:35-42.

34. Haas RH, Nasirian F, Nakano K, et al. Low platelet mitochondrial complex I and complex II/III activity in early untreated Parkinson's disease. Ann Neurol. 1995;37(6):714-722.

35. Gotz ME, Gerstner A, Harth R, et al. Altered redox state of platelet coenzyme Q10 in Parkinson's disease. J Neural Transm. 2000;107(1): 41-48.

36. Sohmiya M, Tanaka M, Tak NW, et al. Redox status of plasma coenzyme Q10 indicates elevated systemic oxidative stress in Parkinson's disease. J Neurol Sci. 2004;223(2):161-166.

37. Isobe $\mathrm{C}, \mathrm{Abe} \mathrm{T}$, Terayama $\mathrm{Y}$. Levels of reduced and oxidized coenzymeQ-10 and 8-hydroxy-2'-deoxyguanosine in the cerebrospinal fluid of patients with living Parkinson's disease demonstrate that mitochondrial oxidative damage and/or oxidative DNA damage contributes to the neurodegenerative process. Neurosci Lett. 2010;469(1): 159-163.

38. Younes-Mhenni S, Frih-Ayed M, Kerkeni A, Bost M, Chazot G Peripheral blood markers of oxidative stress in Parkinson's disease. Eur Neurol. 2007;58(2):78-83.

39. Bogdanov M, Matson WR, Wang L, et al. Metabolomic profiling to develop blood biomarkers for Parkinson's disease. Brain. 2008;131(2): 389-396.

40. Gmitterova K, Heinemann U, Gawinecka J, et al. 8-OHdG in cerebrospinal fluid as a marker of oxidative stress in various neurodegenerative diseases. Neurodegener Dis. 2009;6(5-6):263-269.

41. Ilic TV, Jovanovic M, Jovici AC, Tomovic M. Oxidative stress indicators are elevated in de novo Parkinson's disease patients. Funct Neurol. 1999;14(3):141-147.

42. Seet RC, Lee CY, Lim EC, et al. Oxidative damage in Parkinson disease: measurement using accurate biomarkers. Free Radic Biol Med. 2010;48(4):560-566.

43. Cipriani S, Desjardins CA, Burdett TC, Xu Y, Xu K, Schwarzschild MA. Urate and its transgenic depletion modulate neuronal vulnerability in a cellular model of Parkinson's disease. PLoS One. 2012;7(5):e37331.

44. Gong L, Zhang QL, Zhang N, et al. Neuroprotection by urate on 6-OHDA-lesioned rat model of Parkinson's disease: linking to Akt/ GSK3beta signaling pathway. J Neurochem. 2012;123(5):876-885.

45. Chen X, Burdett TC, Desjardins CA, et al. Disrupted and transgenic urate oxidase alter urate and dopaminergic neurodegeneration. Proc Natl Acad Sci U SA. 2013;110(1):300-305. 
46. Cipriani S, Chen X, Schwarzschild MA. Urate: a novel biomarker of Parkinson's disease risk, diagnosis and prognosis. Biomark Med. 2010;4(5):701-712.

47. Ascherio A, LeWitt PA, Xu K, et al. Urate as a predictor of the rate of clinical decline in Parkinson disease. Arch Neurol. 2009;66(12): $1460-1468$.

48. Schwarzschild MA, Schwid SR, Marek K, et al. Serum urate as a predictor of clinical and radiographic progression in Parkinson disease. Arch Neurol. 2008;65(6):716-723.

49. Johansen KK, Wang L, Aasly JO, et al. Metabolomic profiling in LRRK2-related Parkinson's disease. PLoS One. 2009;4(10):e7551.

50. Rentzos M, Nikolaou C, Andreadou E, et al. Circulating interleukin-15 and RANTES chemokine in Parkinson's disease. Acta Neurol Scand. 2007;116(6):374-379.

51. Dufek M, Hamanova M, Lokaj MJ, et al. Serum inflammatory biomarkers in Parkinson's disease. Parkinsonism Relat Disord. 2009;15(4): 318-320.

52. Mogi M, Harada M, Riederer P, Narabayashi H, Fujita K, Nagatsu T. Tumor necrosis factor-alpha (TNF-alpha) increases both in the brain and in the cerebrospinal fluid from parkinsonian patients. Neurosci Lett. 1994;165(1-2):208-210.

53. McRae-Degueurce A, Klawans HL, Penn RD, et al. An antibody in the CSF of Parkinson's disease patients disappears following adrenal medulla transplantation. Neurosci Lett. 1988;94(1-2):192-197.

54. Double KL, Rowe DB, Carew-Jones FM, et al. Anti-melanin antibodies are increased in sera in Parkinson's disease. Exp Neurol. 2009;217(2): 297-301.

55. Barnum CJ, Tansey MG. Neuroinflammation and non-motor symptoms: the dark passenger of Parkinson's disease? Curr Neurol Neurosci Rep. 2012;12(4):350-358.

56. Rocha NP, Teixeira AL, Scalzo PL, et al. Plasma levels of soluble tumor necrosis factor receptors are associated with cognitive performance in Parkinson's disease. Mov Disord. 2014;29(4):527-531.

57. Lindqvist D, Hall S, Surova Y, et al. Cerebrospinal fluid inflammatory markers in Parkinson's disease - associations with depression, fatigue, and cognitive impairment. Brain Behav Immun. 2013;33: 183-189.

58. Maita C, Tsuji S, Yabe I, et al. Secretion of DJ-1 into the serum of patients with Parkinson's disease. Neurosci Lett. 2008;431(1): 86-89.

59. Waragai M, Nakai M, Wei J, et al. Plasma levels of DJ-1 as a possible marker for progression of sporadic Parkinson's disease. Neurosci Lett. 2007;425(1):18-22.

60. Waragai M, Wei J, Fujita M, et al. Increased level of DJ-1 in the cerebrospinal fluids of sporadic Parkinson's disease. Biochem Biophys Res Commun. 2006;345(3):967-972.

61. Lin X, Cook TJ, Zabetian CP, et al. DJ-1 isoforms in whole blood as potential biomarkers of Parkinson disease. Sci Rep. 2012;2:954.

62. Saito Y, Hamakubo T, Yoshida Y, et al. Preparation and application of monoclonal antibodies against oxidized DJ-1. Significant elevation of oxidized DJ-1 in erythrocytes of early-stage Parkinson disease patients. Neurosci Lett. 2009;465(1):1-5.

63. Rosen C, Hansson O, Blennow K, Zetterberg H. Fluid biomarkers in Alzheimer's disease - current concepts. Mol Neurodegener. 2013;8:20

64. Maarouf CL, Beach TG, Adler CH, et al. Quantitative appraisal of ventricular cerebrospinal fluid biomarkers in neuropathologically diagnosed Parkinson's disease cases lacking Alzheimer's disease pathology. Biomark Insights. 2013;8:19-28.

65. Parkinson Progression Marker Initiative. The Parkinson Progression Marker Initiative (PPMI). Prog Neurobiol. 2011;95(4):629-635.

66. Shoulson I. DATATOP: a decade of neuroprotective inquiry. Parkinson Study Group. Deprenyl and Tocopherol Antioxidative Therapy of Parkinsonism. Ann Neurol. 1998;44(3 Suppl 1):S160-S166.

67. Zhang J, Mattison HA, Liu C, et al. Longitudinal assessment of tau and amyloid beta in cerebrospinal fluid of Parkinson disease. Acta Neuropathol. 2013;126(5):671-682.
68. Leverenz JB, Watson GS, Shofer J, Zabetian CP, Zhang J, Montine TJ. Cerebrospinal fluid biomarkers and cognitive performance in nondemented patients with Parkinson's disease. Parkinsonism Relat Disord. 2011;17(1):61-64.

69. Wang Y, Hancock AM, Bradner J, et al. Complement 3 and factor $\mathrm{h}$ in human cerebrospinal fluid in Parkinson's disease, Alzheimer's disease, and multiple-system atrophy. Am J Pathol. 2011;178(4):1509-1516.

70. Siderowf A, Xie SX, Hurtig H, et al. CSF amyloid \{beta\} 1-42 predicts cognitive decline in Parkinson disease. Neurology. 2010;75(12): $1055-1061$

71. Nicholson JK, Lindon JC. Systems biology: metabonomics. Nature. 2008;455(7216):1054-1056.

72. Di Girolamo F, Lante I, Muraca M, Putignani L. The role of mass spectrometry in the "omics" era. Curr Org Chem. 2013;17(23):2891-2905.

73. Caudle WM, Bammler TK, Lin Y, Pan S, Zhang J. Using 'omics' to define pathogenesis and biomarkers of Parkinson's disease. Expert Rev Neurother. 2010;10(6):925-942.

74. Grunblatt E, Mandel S, Jacob-Hirsch J, et al. Gene expression profiling of parkinsonian substantia nigra pars compacta; alterations in ubiquitinproteasome, heat shock protein, iron and oxidative stress regulated proteins, cell adhesion/cellular matrix and vesicle trafficking genes. J Neural Transm. 2004;111(12):1543-1573.

75. Moran LB, Graeber MB. Towards a pathway definition of Parkinson's disease: a complex disorder with links to cancer, diabetes and inflammation. Neurogenetics. 2008;9(1):1-13.

76. Sutherland GT, Matigian NA, Chalk AM, et al. A cross-study transcriptional analysis of Parkinson's disease. PLoS One. 2009;4(3):e4955.

77. Grunblatt E, Zehetmayer S, Jacob CP, Müller T, Jost WH, Riederer P. Pilot study: peripheral biomarkers for diagnosing sporadic Parkinson's disease. J Neural Transm. 2010;117(12):1387-1393.

78. Scherzer CR, Eklund AC, Morse LJ, et al. Molecular markers of early Parkinson's disease based on gene expression in blood. Proc Natl Acad Sci U SA. 2007;104(3):955-960.

79. Molochnikov L, Rabey JM, Dobronevsky E, et al. A molecular signature in blood identifies early Parkinson's disease. Mol Neurodegener. 2012;7:26.

80. Basso M, Giraudo S, Corpillo D, Bergamasco B, Lopiano L, Fasano M. Proteome analysis of human substantia nigra in Parkinson's disease. Proteomics. 2004;4(12):3943-3952.

81. Werner CJ, Heyny-von Haussen R, Mall G, Wolf S. Proteome analysis of human substantia nigra in Parkinson's disease. Proteome Sci. 2008;6:8

82. Abdi F, Quinn JF, Jankovic J, et al. Detection of biomarkers with a multiplex quantitative proteomic platform in cerebrospinal fluid of patients with neurodegenerative disorders. JAlzheimers Dis. 2006;9(3): 293-348.

83. Constantinescu R, Andreasson U, Li S, et al. Proteomic profiling of cerebrospinal fluid in parkinsonian disorders. Parkinsonism Relat Disord. 2010;16(8):545-549.

84. Biomarkers Definitions Working Group. Biomarkers and surrogate endpoints: preferred definitions and conceptual framework. Clin Pharmacol Ther. 2001;69(3):89-95.

85. Jin J, Hulette C, Wang Y, et al. Proteomic identification of a stress protein, mortalin/mthsp70/GRP75: relevance to Parkinson disease. Mol Cell Proteomics. 2006;5(7):1193-1204.

86. Pan S, Rush J, Peskind ER, et al. Application of targeted quantitative proteomics analysis in human cerebrospinal fluid using a liquid chromatography matrix-assisted laser desorption/ionization time-offlight tandem mass spectrometer (LC MALDI TOF/TOF) platform. J Proteome Res. 2008;7(2):720-730.

87. Li YH, Wang J, Zheng XL, et al. Matrix-assisted laser desorption/ ionization time-of-flight mass spectrometry combined with magnetic beads for detecting serum protein biomarkers in Parkinson's disease. Eur Neurol. 2011;65(2):105-111.

88. Zhao X, Xiao WZ, Pu XP, Zhong LJ. Proteome analysis of the sera from Chinese Parkinson's disease patients. Neurosci Lett. 2010;479(2): $175-179$. 
89. Mila S, Albo AG, Corpillo D, et al. Lymphocyte proteomics of Parkinson's disease patients reveals cytoskeletal protein dysregulation and oxidative stress. Biomark Med. 2009;3(2):117-128.

90. Mapstone M, Cheema AK, Fiandaca MS, et al. Plasma phospholipids identify antecedent memory imipairments in older adults. Nat Med. 2014;20(4):415-418.

91. LeWitt P. Recent advances in CSF biomarkers for Parkinson's disease. Parkinsonism Relat Disord. 2012;18 Suppl 1:S49-S51.

92. Vallelunga A, Ragusa M, Di Mauro S, et al. Identification of circulating micro-RNAs for the differential diagnosis of Parkinson's disease and multiple system atrophy. Front Cell Neurosci. 2014;8:156.
93. Botta-Orfila T, Morato X, Compta Y, et al. Identification of blood serum micro-RNAs associated with idiopathic and LRRK2 Parkinson's disease. J Neurosci Res. 2014;92(8):1071-1077.

94. The Parkinson Study Group. Inosine to increase serum and cerebrospinal fluid urate in Parkinson disease: a randomized clinical trial. JAMA Neurol. 2013;71(2):141-150.

95. Kang UJ, Alcalay R, Goldman JM, et al. The BioFIND study (Fox Investigation for New Discovery of Biomarkers in Parkinson's disease): design and methodology. Neurology. 2014;10 Suppl:P4.043.

\section{Publish your work in this journal}

Current Biomarker Findings is an international, peer-reviewed, open access journal publishing original research, reports, reviews and commentaries on all areas of biomarker research. The manuscript management system is completely online and includes a very quick and fair

\section{Dovepress}

peer-review system. Visit http://www.dovepress.com/testimonials.php to read real quotes from published authors. 